positive inflammatory assessment with very high activity indices, with a mean of 4.6. $64.66 \%$ of the patients received NSAIDs, of which $11 \%$ responded well. $57 \%$ were treated with csDMARDs, and $17.86 \%$ were treated with biologics. At the time of our study, the mean visual analog scale was $5.84 \pm 1.7$ out of $10(2-9)$. The mean Epworth score was $8.38 \pm 5.2(0-21) .56 .1 \%$ of patients had no sleep debt, $33.3 \%$ had a sleep deficit, and only $10.6 \%$ had signs of drowsiness. For the overall Pittsburgh score, the mean was $7.02 \pm 3.6(1-18)$. The mean of "subjective quality of sleep" was 1.12 , "sleep latency" was 1.22, "duration of sleep" was 1.06, "usual sleep efficiency" was 0.74 , "Sleep disturbance" of 1.28 , "use of a sleep medication" of 0.54 , and the average of the component concerning "poor shape during the day" was 1.03 out of 3 . The LEQUESNE index went from an average of 6 to 8 , which corresponds to an average handicap $(P=0.2)$ over a period of 3 years. $68 \%$ of the patients had an alteration in the quality of sleep, starting on average three years after the onset of symptoms. $11 \%$ reported having experiencedanxiety and depressive symptoms, and reported having used antidepressants or anxiolytics in the past 5 years.

Conclusion: Our study showed the negative impact of SpA on the duration and overall quality of sleep. The degree of pain as well as functional impairment can cause and worsen sleep disturbances in SpA. We have shown that the Pittsburg score increases significantly with the increase of pain. The Lequesne score and that the Epworth score increase with disease activity[1].

REFERENCES:

[1] StolwijkC,vanTubergenA,Castillo-OrtizJD,BoonenA.Prevalenceofextra-articularmanifestationsinpatientswithankylosingspondylitis:asystematicreviewandmeta-analysis.AnnRheumDis2015;74:65-73.

Disclosure of Interests: None declared.

DOI: 10.1136/annrheumdis-2021-eular.2909

\section{POS0999 HIGH CORRELATIONS BETWEEN QUESTION 1 \& 2 OF THE BASFI: AN OPPORTUNITY TO STREAMLINE THE BASFI}

M. Maclean ${ }^{1}$, S. Maguire ${ }^{1}$, F. B. O'shea'. ' St. James' Hospital, Rheumatology, Dublin, Ireland

Background: The Bath Ankylosing Spondylitis Functional Index (BASFI) is a useful tool to quantitatively characterise functional ability in a patient with axial spondyloarthropathy (axSpA). Whether first presentation, decompensated, or routine follow-up, BASFI can help establish a more accurate understanding of disease progression, or response. As with any questionnaire, relevance and absence of redundancy is required. This analysis questions the redundancy of the first two questions of the BASFI:

1) How difficult is it to put socks on your feet?

2) How difficult is it to pick a pen up off the floor?

Objectives: To compare variation in reporting of questions one and two of the BASFI, to establish redundancy or exclusivity of these questions.

Methods: IBM SPSS version 26 was used for data analysis. Data from axSpA patients who attended the Rheumatology department during the study period were included in the analysis where BASFI scores were available. Both variables (pen scores and sock scores) were assessed with a Shapiro-Wilk's test for normal distribution. The variables were also assessed for the presence of a monotonic relationship by visual inspection of a scatterplot of the variables. Once a monotonic relationship was established a Spearman's rank order correlation between the sock score and pen scores for each participant was analysed.

Results: In total data from 82 axSpA patients were included in this analysis. Population was made up of $28 \%$ (23) females, $72 \%$ (59) males with mean BASDAI score 4.33 and mean BASFI score 3.88 (Table 1). Both variables were not normally distributed as assessed by Shapiro-Wilk's test $(p<0.05)$ necessitating a Spearman's rank-order correlation for analysis. Preliminary analysis variables demonstrated the relationship between the variables to be monotonic as determined by visual inspection of the scatterplot (graph 1) with no outliers detected. There was a statistically significant, strong positive correlation between sock scores and pen scores in this axSpA population, $r_{\text {partial }}(80)=0.809$ significant at the $p<0.01$ level.

Table 1. Descriptive output of data

\begin{tabular}{ll}
\hline & $\%(\mathbf{n})$ \\
\hline $\mathbf{n}$ & 82 \\
Females & $28 \%(23)$ \\
Males & $72 \%(59)$ \\
Age & 45.03 \\
BASDAI & 4.33 \\
BASFI & 3.88 \\
Pen score & 3.93 \\
Sock score & 2.88 \\
Sock score greater & $7.3 \%(6)$ \\
Pen score greater & $50 \%(41)$ \\
Same scores & $42.7 \%(35)$ \\
\hline
\end{tabular}

Conclusion: There is a strong positive correlation between sock (question 1) and pen scores (question 2) as captured by the BASFI. It appears that both questions are capturing a similar functional limitation in patients with axSpA. In order to minimise redundancy and improve the relevance of the BASFI our results support the removal of one of these questions to simplify the BASFI. From a practical perspective, putting on socks (question 1) would be a more commonly encountered daily activity than picking up a

pen from the floor(question 2). As such, we would suggest removal of question 2 from the BASFI.

Table 1 \& Figure 1

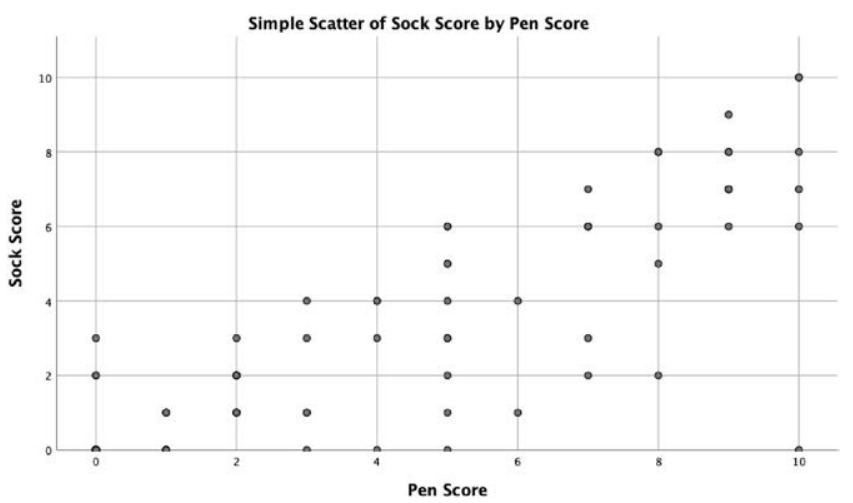

Graph 1. Spread of Data points demonstrating a monotonic relationship with no outliers

Disclosure of Interests: Michael MacLean: None declared, Sinead Maguire Grant/research support from: Recipient of the Gilead Inflammation Fellowship, Finbar Barry O'Shea: None declared.

DOI: 10.1136/annrheumdis-2021-eular.2913

\section{\begin{tabular}{|l|l}
\hline POS1000 COMORBIDITIES ASSOCIATED TO \\
\hline
\end{tabular} SPONDYLOARTHRITIS}

S. Bouden ${ }^{1}$, L. Kharrat ${ }^{1}$, A. Ben Tekaya ${ }^{1}$, O. Saidane ${ }^{1}$, R. Tekaya ${ }^{1}$, I. Mahmoud ${ }^{1}$, L. Abdelmoula'. ' Hospital Charles Nicolle, Rheumatology, Tunis, Tunisia

Background: In contrast to other chronic rheumatic diseases such as rheumatoid arthritis, comorbidities associated to spondyloarthritis (SpA) and their impact on disease outcomes are less well studied.

Objectives: The aim of our study was to investigate the prevalence of comorbidities among SpA patients and to determine factors influencing their appearance. Methods: We conducted a retrospective study including patients meeting the Assessment of SpondyloArthritis International Society (ASAS) criteria between 2000 and 2020.

The following comorbidities were collected: cardiovascular pathologies and their risk factors (smoking, arterial hypertension, diabetes, dyslipidemia and obesity), neoplasms, osteoporosis, depression, infections, gastrointestinal and pulmonary disorders. Results: We included 138 patients. Sixty-eight per cent of them were males. The mean age was $45.73 \pm 12.66$ years. The mean age at the disease onset was $28.89 \pm 12.54$ years. The mean CRP was $33.38 \pm 39.65 \mathrm{mg} / \mathrm{dL}$. The mean BASDAI and ASDAS-CRP were $4.21 \pm 2.23$ and $3.06 \pm 1.26$, respectively. The mean BASFI was $4.77 \pm 2.58$.

Sixty patients had at least one comorbidity (43.5\%): 53 patients had one comorbidity (38.4\%), 21 accumulated two types of comorbidities (15.2\%) and 7 patients accumulated three types or more (5\%).

Osteoporosis was the most frequent comorbidity, it was present in $23.1 \%$ of the cases $(n=32)$, followed by tuberculosis $8.7 \%(n=12)$, stomach ulcers $5.1 \%(n=7)$, pulmonary superinfection $2.9 \%(n=4)$, neoplasia $2.2 \%(n=3)$ and then depression $1.4 \%(n=2)$.

Cardiovascular risk factors were noted in 44 patients $(31.9 \%)$ : hypertension $(15.9 \%)$, diabetes $(12.3 \%)$, dyslipidemia $(9.4 \%)$ and obesity $(8.7 \%)$.

Thirty-seven per cent of our patients were smokers.

$\mathrm{SpA}$ patients with comorbidities were significantly older than those without $(50.2 \pm 11.07$ versus $42.3 \pm 12.8$ years, $p<0.0001)$.

The presence of comorbidities was significantly associated to a higher disease activity evaluated by BASDAI $(p=0.005)$ and ASDAS-CRP $(p=0.002)$. Furthermore, BASFI was significantly higher among patients with comorbidities (5.47 \pm 2.38 versus $4.31 \pm 2.62, p=0.028)$.

However, no association was found between presence of comorbidities and smocking or CRP.

Conclusion: Our results show that more than $40 \%$ of our SpA patients presented with at least one comorbidity. Remarkably, the presence of comorbidities 
was associated with high disease activity, suggesting that that inflammation might promotes comorbidities. For optimal management of SpA, a systemic screening for comorbidities is essential.

Disclosure of Interests: None declared.

DOI: 10.1136/annrheumdis-2021-eular.2929

\section{POS1001 $\quad$ CLINICAL AND SURROGATE CARDIOVASCULAR RISK ASSESSMENT AND ITS RELATIONSHIP WITH PSORIATIC ARTHRITIS PATHOGENESIS}

N. Barbarroja Puerto ${ }^{1}$, I. Arias de la Rosa ${ }^{1}$, C. Román-Rodriguez ${ }^{2}$, I. Gómez García $^{2}$, C. Perez-Sanchez ${ }^{2}$, C. López-Medina², M. L. Ladehesa Pineda ${ }^{2}$, D. Ruiz ${ }^{2}$, A. M. Patiño-Trives ${ }^{2}$, M. Luque-Tévar² ${ }^{2}$, C. Lopez-Pedrera ${ }^{2}$, A. Escudero Contreras $^{2}$, E. Collantes Estevez ${ }^{2}$, M. D. López Montilla ${ }^{2} .{ }^{1}$ Maimonides Institute for Research in Biomedicine of Cordoba (IMIBIC)/Reina Sofia Hospital/ University of Cordoba, Medicine Department, Cordoba, Spain; ${ }^{2}$ Maimonides Institute for Research in Biomedicine of Cordoba (IMIBIC)/Reina Sofia Hospital/University of Cordoba, Rheumatology Department, Cordoba, Spain

Background: Psoriatic arthritis (PsA) is a chronic inflammatory disease associated with an increased prevalence of cardiovascular (CV) events. Traditional CV risk factors do not account for the increased CV disease mortality in PsA. Inflammation seems to have a key role in the development of this comorbidity, however the specific molecular mechanisms involved are not defined yet.

Objectives: To evaluate clinical CV risk factors and surrogate markers and their relationship with inflammation, disease activity and metabolic comorbidities in PsA patients

Methods: This is a cross-sectional study including 100 PsA patients without CV disease recruited in the routine clinical practice at the Rheumatology Department, Reina Sofia Hospital of Cordoba and 100 age-matched healthy donors (HDs). Different parameters related to the cardiometabolic risk were analyzed. Clinical and analytical parameters were collected: lipid profile (cholesterol, HDL, LDL, TG, ApoA and ApoB), glucose and insulin, body surface area (BSA) affected by psoriasis, number of tender and swollen joints, DAPSA, VAS, CRP and ESR. To measure the persistence of inflammation, CRP levels were recorded retrospectively once, twice, or three times during the 5 years prior to study and at the moment of the study. Increased levels of CRP in at least $50 \%$ of the determinations was considered as persistent inflammation. Plasma and peripheral blood mononuclear cells (PBMCs) were isolated from peripheral blood of patients and HDs. A panel of 92 proteins involved in CV disease and an adipocytokine profile was measured in plasma and PBMCs. In addition, activation of 18 intracellular pathways involved in cell activation was also measured in PBMCs. In vitro experiments in adipocytes treated with serum from PsA patients were also carried out. Results: Traditional CV risk factors including atherogenic risk, insulin resistance (IR), metabolic syndrome, smoking, obesity, arterial hypertension, apolipoprotein B/A risk, type 2 diabetes mellitus and the levels of SCORE were significantly increased in PsA patients. The presence of IR was associated with disease activity markers (DAPSA, ESR and CRP). In fact, the HOMA-IR index was related to the CRP persistence. PsA patients with obesity had significantly increased the number of tender and swollen joints, the levels of DAPSA and CRP. Twenty-eight proteins involved in CV disease and six adipocytokines were significantly elevated in the plasma of PsA patients. Several of these cardiovascular molecules were associated with higher levels of DAPSA (CTSD, GAL3, CD163, FABP4, IL6 and IL1RT2), acute phase reactants (GAL3, TNF $\alpha$, Adiponectin, TNFR1 and IL6), affected body surface area (IL2RA, GAL3, CCL15, TRAP, CSTB, CD163, OPG and CNTN1) and onychopaty (TRAP, VWF, MCP-1, GAL3, LTBR, TFPI, CHI3L1, CTSZ and JAM-A). In addition, the mRNA expression of most of those $28 \mathrm{CV}$ molecules were significantly increased in PBMCs from PSA patients. At intracellular level, the activation of 11 kinases (ERK1/2, AKT, S6 Ribosomal, mTOR, HSP27, Bad, p70 S6 kinase, PRAS40, p53 and caspase-3) involved in insulin signaling, inflammation, cell survival and apoptosis were altered in PBMCs. Finally, serum from PsA patients was able to modify the expression of these molecules in adipocytes. Conclusion: 1) Disease activity and inflammatory burden are closely associated with the presence of metabolic alterations, specifically obesity and IR in patients with PsA. 2) The development of IR is extremely related to the persistence of CRP levels in the previous 5 years. 3 ) Inflammation is closely associated to the adipose tissue dysfunction in PSA and 4) FABP4, CD163 and GAL3 are surrogate $\mathrm{CV}$ markers commonly associated with clinical features of PsA, suggesting the role of these molecules linking CVD and PsA pathogenesis.

Funded by ISCIII (PI17/01316 and RIER RD16/0012/0015) co-funded with FEDER.

Disclosure of Interests: None declared.

DOI: 10.1136/annrheumdis-2021-eular.2990

\section{POS1002 \\ BASELINE CALPROTECTIN AND VISFATIN LEVELS PREDICT RADIOGRAPHIC SPINAL PROGRESSION AFTER 2 YEARS IN ANKYLOSING SPONDYLITIS PATIENTS ON TNF INHIBITOR THERAPY}

J. Rademacher ${ }^{1,2}$, M. Siderius ${ }^{3}$, L. Gellert ${ }^{1}$, F. Wink ${ }^{4}$, M. Verba $^{1}$, F. Maas ${ }^{3}$, L. M. Tietz ${ }^{1}$, D. Poddubnyy ${ }^{1,5}$, A. Spoorenberg ${ }^{3}$, S. Arends ${ }^{3} .{ }^{1}$ Charité - $^{-}$ Universitätsmedizin Berlin, Department of Gastroenterology, Infectiology and Rheumatology, Berlin, Germany; ${ }^{2}$ Berlin Institute of Health, BIH, Berlin, Germany; ${ }^{3}$ University Medical Center Groningen, Department of Rheumatology and Clinical Immunology, Groningen, Netherlands; ${ }^{4}$ Medical Center Leeuwarden, Department of Rheumatology, Leeuwarden, Netherlands ${ }^{5}$ Deutsches Rheuma-Forschungszentrum Berlin (DRFZ), ein Institut der Leibniz-Gemeinschaft, Epidemiology, Berlin, Germany

Background: Radiographic spinal progression determinates functional status and mobility in ankylosing spondylitis (AS) ${ }^{1}$.

Objectives: To analyse whether biomarker of inflammation, bone turnover and adipokines at baseline or their change after 3 months or 2 years can predict spinal radiographic progression after 2 years in AS patients treated with TNF- $\alpha$ inhibitors (TNFi).

Methods: Consecutive AS patients from the Groningen Leeuwarden Axial Spondyloarthritis (GLAS) cohort ${ }^{2}$ starting TNFi between 2004 and 2012 were included. The following serum biomarkers were measured at baseline, 3 months and 2 years of follow-up with ELISA:

- Markers of inflammation: calprotectin, matrix metalloproteinase-3 (MMP-3), vascular endothelial growth factor (VEGF)

- Markers of bone turnover: bone-specific alkaline phosphatase (BALP), serum C-terminal telopeptide (SCTX), osteocalcin (OC), osteoprotegerin (OPG), procollagen typ I and II N-terminal propeptide (PINP; PIINP), sclerostin.

Adipokines: high molecular weight (HMW) adiponectin, leptin, visfatin Two independent readers assessed spinal radiographs at baseline and 2 years of follow-up according to the modified Stoke Ankylosing Spondylitis Spine Score (mSASSS). Radiographic spinal progression was defined as mSASSS change $\geq 2$ units or the formation of $\geq 1$ new syndesmophyte over 2 years. Logistic regression was performed to examine the association between biomarker values at baseline, their change after 3 months and 2 years and radiographic spinal progression. Multivariable models for each biomarker were adjusted for mSASSS or syndesmophytes at baseline, elevated CRP ( $\geq 5 \mathrm{mg} / \mathrm{l}$ ), smoking status, male gender, symptom duration, $\mathrm{BMI}$, and baseline biomarker level (the latter only in models with biomarker change).

Results: Of the 137 included AS patients, $72 \%$ were male, $79 \%$ HLAB27+; mean age at baseline was 42 years (SD 10.8), ASDAScrp $3.8(0.8)$ and mSASSS 10.6 (16.1). After 2 years of follow-up, 33\% showed mSASSS change $\geq 2$ units and $24 \%$ had developed $\geq 1$ new syndesmophyte. Serum levels of biomarkers of inflammation and bone formation showed significant changes under TNFi therapy, whereas adipokine levels were not altered from baseline (Figure 1).

Univariable logistic regression revealed a significant association of baseline visfatin (odds ratio OR [95\% confidence interval] 1.106 [1.007-1.215]) and sclerostin serum levels (OR 1.006 [1.001-1.011]) with mSASSS progression after 2 years. Baseline sclerostin levels were also associated with syndesmophyte progression (OR 1.007 [1.001-1.013]). In multivariable logistic analysis, only baseline visfatin level remained significantly associated (OR 1.465 [1.137-1.889]) with mSASSS progression. Furthermore, baseline calprotectin showed a positive association with both, mSASSS (OR 1.195 [1.055-1.355]) and syndesmophyte progression (OR 1.107 [1.001-1.225]) when adjusting for known risk factors for radiographic progression.

Univariable logistic regression showed that change of sclerostin after 3 months was associated with syndesmophytes progression (OR 1.007 [1.000-1.015), change of PINP level after 2 years was associated with mSASSS progression (OR 1.027 [1.003-1.052]) and change of visfatin after 2 years was associated with both measures of radiographic progression - mSASSS (OR 1.108 [1.004-1.224]) and syndesmophyte formation (OR 1.115; [1.002-1.24]). However, those associations were lost in multivariable analysis.

Conclusion: Independent of known risk factors, baseline calprotectin and visfatin levels were associated with radiographic spinal progression after 2 years of TNFi. Although biomarkers of inflammation and bone formation showed significant changes under TNFi therapy, these changes were not significantly related to radiographic spinal progression in our cohort of AS patients.

\section{REFERENCES:}

[1] Poddubnyy et al 2018

[2] Maas et al 2019 